\title{
REVISTA DA FACULDADE DE ODONTOLOGIA DE PORTO ALEGRE
}

JOURNAL OF SCHOOL OF DENTISTRY OF PORTO ALEGRE

REITOR DA UFRGS/RECTOR OF UFRGS

Prof. Dr. Carlos Alexandre Neto

VICE-REITOR/VICE RECTOR

Prof. Dr. Rui Vicente Oppermann

DIRETOR DA FACULDADE DE ODONTOLOGIA

DEAN OF THE SCHOOL OF DENTISTRY

Prof. Dr. Pantelis Varvaki Rados

VICE-DIRETOR DA FACULDADE DE ODONTOLOGIA

VICE DEAN OF THE SCHOOL OF DENTISTRY

Prof. Régis dos Santos Burmeister

COMISSÃO EDITORIAL/ EDITORIAL COMMITTEE

Editor Científico/ Scientific Editor

Prof. Dr. Cassiano Kuchenbecker Rösing

Editores Associados/ Associate Editors

Prof. Dr. Alex Nogueira Haas

Profa. Dra. Altair Antoninha Del-Bel Cury

Profa. Dra. Fabiana Soares Grecca

\section{CONSELHO EDITORIAL/EDITORIAL BOARD}

Prof. Dr. Alberto Consolaro (FOB-USP)

Profa. Dra. Ana Maria Sphor (FO-PUCRS)

Prof. Dr. André Francisco H. Ferreira (Univ. of Alabama, USA)

Profa. Dra. Anna Christina M. Fossati (ICBS-UFRGS)

Profa. Dra. Anne Merete Aass (Univ. De Oslo, NORUEGA)

Prof. Dr. Antônio de L. L. Costa (UFRN)

Prof. Dr. Benjamin Martinez (Univ. do Chile)

Profa. Dra. Cínthia P. M. Tabchoury (FOP-UNICAMP)

Profa. Dra. Claídes Abegg (FO-UFRGS/FO-ULBRA)

Prof. Dr. Cristiano Susin (Univ. Geórgia, USA)

Profa. Dra. Dalva Maria P. Padilha (FO-UFRGS)

Profa. Dra. Dione D. Torriani (FO-UFPEL)

Prof. Dr. Ernani M. Marchioro (FO-PUCRS/FO-ULBRA)

Profa. Dra. Edela Puricelli (FO-UFRGS)

Prof. Dr. Eduardo Mondragon (Univrsity of Florida, USA)

Prof. Dr. Fernando B. de Araújo (FO-UFRGS)

Prof. Dr. Fernando Neves Hugo (FO-UFRGS)

Prof. Dr. Fernando H. Westphalen (FO-UFPR/FO-PUCPR)

Prof. Dr. Guilherme C. Lopes (CCS-UFSC)

Profa. Dra. Heloisa Emília Dias da Silveira (FO-UFRGS)

Prof. Dr. Hugo M. S. Oshima (FO-PUCRS) 
Profa. Dra. Joana Carvalho (Catholic University of LOUVAIN, Bélgica)

Prof. Dr. Jacques Eduardo Nor (Univ. of Michigan, USA)

Prof. Dr. José Antônio Poli Figueiredo (FO-UFRGS)

Prof. Dr. Manoel Sant'Ana Filho (FO-UFRGS)

Prof. Dr. Márcio de L. Grossi (FO-PUCRS)

Profa. Dra. Maria de Lourdes de A. Massara (FO-UFMG)

Prof. Dr. Mario Fernando de Góes (FOP-UNICAMP)

Prof. Dr. Mário Tanomaru Filho (FOAR-UNESP)

Profa. Dra. Marinilce F. dos Santos (FO-USP)

Profa. Dra. Marisa Maltz (FO-UFRGS)

Profa. Dra. Martha Siragusa (Univ. Nacional Rosário, ARG)

Prof. Dr. Pantelis V. Rados (FO-UFRGS)

Profa. Dra. Patrícia dos S. Jardim (FO-UFPel)

Profa. Dra. Rejane F. Ribeiro Rotta (UFG)

Prof. Dr. Rodivan B. da Cunha (FOP-UPE)

Profa. Dra. Rosemary S. A. Shinkai (FO-PUCRS)

Prof. Dr. Rui Vicente Oppermann (FO-UFRGS)

Prof. Dr. Simonides Consani (FOP-UNICAMP)

Profa. Dra. Sonia Maria B. de Slavutzky (FO-UFRGS)

Profa. Dra. Susana Maria W. Samuel (FO-UFRGS)

Profa. Dra. Vania Regina Camargo Fontanella

\section{BIBLIOTECÁRIAS/LIBRARIANS}

Ida Rossi-CRB 10/586

Rejane Raffo Klaes - CRB 10/771

\section{INDEXAÇÃO/INDEXING}

Revista da Faculdade de Odontologia de Porto Alegre é publicada a cada 4 meses e está indexada na BBO (Biblioteca Brasileira de Odontologia) desde 1982 e na LILACS (Literatura Latino Americana e do Caribe de Informação em Ciências da Saúde) desde 1982.

The Journal of School of Dentistry of Porto Alegre is published every 6 months and is indexed in BBO and LILACS since 1982. LAPTOC - Latin American Periodicals Table of Contents since 2002.

Editoração: Derek Cabral

Revisão: Derek Cabral, Alex Nogueira Haas

\section{ENDEREÇO PARA CORRESPONDÊNCIA CORRESPONDING ADDRESS}

Revista da Faculdade de Odontologia de Porto Alegre

Rua Ramiro Barcelos, 2492 - CEP 90035-003

Porto Alegre - RS - BRASIL

Fone: + $55513308-5318$

Fax: + $55513308-5010$

E.mail: odonto_revista@ufrgs.br

ISSN: 0566 - 1854 / e-ISSN: 2177-0018 


\section{EDITORIAL}

Ao nos aproximarmos do final deste ano queremos agradecer a toda comunidade da Faculdade por ter indicado a mim juntamente com o Prof. Regis Burmeister do Santos para dirigirmos a Faculdade nos próximos quatro ano.

Sabemos que os compromissos assumidos são inúmeros e sem dúvida o mais desafiador será a implementação do curso noturno em Odontologia.

Esta experiência é inovadora no sistema de ensino público federal superior e está voltada a abrir espaço para a formação do estudante trabalhador. Esta proposta está inserida dentro do projeto REUNI da UFRGS cujo objetivo maior é o da expansão das vagas de graduação nas IFES.

Estes e outros objetivos serão alcançados com a participação de toda a nossa comunidade e com a ajuda da nova administração central da UFRGS recentemente empossada, liderada pelos professores Carlos Alexandre Netto e Rui Vicente Oppermann respectivamente Reitor e Vice Reitor da UFRGS, recentemente eleitos

Prof. Pantelis Varvaki Rados

Diretor da Faculdade de Odontologia da UFRGS 